\title{
suCAQR: A Simplified Communication-Avoiding QR Factorization Solver Using the TBLAS Framework
}

\author{
Weijian Zheng and Fengguang Song \\ Department of Computer Science \\ Indiana University Purdue University Indianapolis \\ Email: \{wz26, fgsong $\} @$ iupui.edu
}

\author{
Lan Lin \\ Department of Computer Science \\ Ball State University \\ Email: 1lin4@ bsu.edu
}

\author{
Zizhong Chen \\ Department of Computer Science \\ University of California at Riverside \\ Email: chen@cs.ucr.edu
}

\begin{abstract}
The scope of this paper is to design and implement a scalable QR factorization solver that can deliver the fastest performance for tall and skinny matrices and square matrices on modern supercomputers. The new solver, named scalable universal communication-avoiding $Q R$ factorization ( $s u C A Q R$ ), introduces a simplified and tuning-less way to realize the communicationavoiding QR factorization algorithm to support matrices of any shapes. The software design includes a mixed usage of physical and logical data layouts, a simplified method of dynamic-root binary-tree reduction, a dynamic dataflow implementation, and an analytical model to determine an optimal number of factorization domains. Compared with the existing communication avoiding $Q R$ factorization implementations, $s u C A Q R$ has the benefits of being simpler, more general, and more efficient. By balancing the degree of parallelism and the proportion of faster computational kernels, it is able to achieve scalable performance on clusters of multicore nodes. The software essentially combines the strengths of both synchronization-reducing approach and communication-avoiding approach to achieve high performance. Based on the experimental results using 1,024 CPU cores, $s u C A Q R$ is faster than DPLASMA by up to $30 \%$, and faster than ScaLAPACK by up to 30 times.

Index Terms-high performance computing; computational science application; performance modeling and optimization; dataflow runtime system.
\end{abstract}

\section{INTRODUCTION}

QR factorization is a fundamental computational kernel for many important scientific, engineering, and big data analytics applications. It has been applied to solving linear systems, least-squares problems, linear regression problems, and the production function modeling, as well as assessing the conditioning of these problems [1]-[3]. The QR factorization of a matrix $A$ of dimension $m \times n(m \geq n)$ takes the form of $A=Q R$, where $Q$ is an $m \times m$ orthogonal matrix, and $R$ $\left(=Q^{T} A\right)$ is an upper triangular matrix with zeros below its diagonal. The design and implementation of more scalable QR factorizations will accelerate a wide range of domain applications.

The most widely used parallel algorithm to solve $\mathrm{QR}$ factorizations is the block $Q R$ factorization algorithm used by the de facto standard ScaLAPACK library [4], [5]. As displayed in Figure 1, matrix $A$ is divided into a thin panel (i.e., $\frac{A_{11}}{A_{21}}$ ) of dimension $M \times \mathrm{NB}$, a block of rows $A_{12}$, and

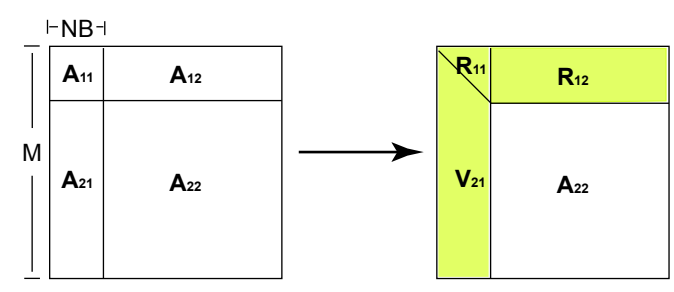

Fig. 1. The classic block QR factorization algorithm used by ScaLAPACK.

a trailing submatrix $A_{22}$. The block algorithm first applies level 1 PBLAS subroutines to the panel $\left(\frac{A_{11}}{A_{21}}\right)$, next it forms the triangular factor from the panel, finally it uses level 3 PBLAS to factor $A_{12}$ and update $A_{22}$. However, since the panel is computed one column after another-resulting in a large communication overhead and surface-to-volume ratio the block algorithm does not scale well for tall and skinny matrices (i.e., matrices with much more rows than columns).

To reduce the large communication overhead with tall and skinny matrices, Demmel et al. then designed an algorithm called Communication-Avoiding QR factorization (CAQR) [6][8]. As explained in Figure 2, instead of computing a sequence of column-by-column operations, CAQR can perform a set of level 3 BLAS operations in the panel. Then it merges the output of the level 3 BLAS operations to get the final factor $R$. Not only does the algorithm convert level 1 BLAS to level 3 BLAS, but also it significantly reduces the number of communication messages. The original work of Demmel et al. [6] offered an estimated performance speedup of CAQR. Later, Song et al. [9] developed the first parallel implementation of CAQR, which is referred to as distributed tiled CAQR [9].

While the distributed tiled CAQR demonstrates good strong scalability and weak scalability on different HPC systems, it only attains good performance for tall and skinny matrices. The issue is that its performance starts to decrease when the input matrix goes from skinny to square. The distributed tiled CAQR algorithm uses a static block distribution method to map blocks of rows to different processes. Although the block distribution method can minimize the inter-node communica- 
tion, it makes certain processes turn into idle processes on nonskinny matrices (see more details in Section II). Another work of Hierarchical QR [10] investigates the effect of using a wide variety of reduction trees to improve the $\mathrm{CAQR}$ performance. However, determining the optimal tree and the optimal number of CAQR domains is dependent on the actual input and the number of CPUs, which are not known until executing the program. In this paper, we target designing a new solver that has a simpler design and implementation, does not require a lot of parameter tuning, and scales well on matrices of any shapes, ranging from extremely tall and skinny to square matrices.

To achieve the goal, we design a QR factorization solver called "Scalable Universal Communication-Avoiding QR Factorization" (suCAQR). The $s u C A Q R$ solver consists of two types of computations: 1) process-local computation, and 2) global binary-tree reduction among all processes. It also mixes two types of data layout: 1) physical block data layout, and 2) logical block-cyclic data layout. The suCAQR employs a logic block cyclic data distribution method to achieve load balancing while applying a tiled QR factorization method to the physical data storage directly to avoid communication. It uses a dynamic-root binary-tree to merge results from different factorization domains (domain is a group of computational tasks that is independent of other groups). Also, suCAQR can maintain a good tradeoff between the degree of parallelism and the number of faster kernels for matrices of different shapes by changing the number of factorization domains. An analytical model is developed to determine the optimal number of factorization domains without any tuning work. Furthermore, suCAQR uses a dynamic dataflow-driven TBLAS runtime [11] to enable efficient synchronization-reducing executions on a specific communication-avoiding algorithm.

We measure and evaluate the weak scalability for three different libraries: ScaLAPACK, DPLASMA [10], [12], and $s u C A Q R$. We also test different matrix shapes ranging from extremely tall and skinny to square using 1,024 cores. Based on the experimental results, $s u C A Q R$ can outperform ScaLAPACK by 30 times and DPLASMA by $30 \%$. Based on the runtime efficiency analysis, we also find that $s u C A Q R$ 's total wall clock execution time is almost equal to its computation time (i.e., the CPU time taken by the computational kernels). This implies that there is no CPU idle time and the communication cost is totally hidden by computations (further discussed in Section VII).

To the best of our knowledge, this work makes the following

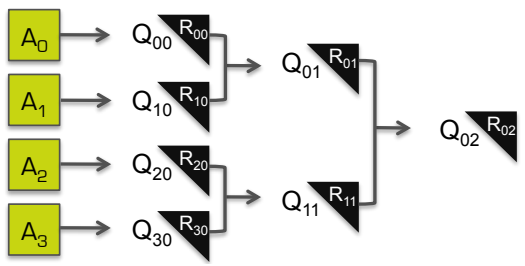

Fig. 2. Communication-Avoiding QR (CAQR) performs level 3 BLAS on the panel (i.e., $A_{0}, A_{1}, A_{2}, A_{3}$ ) followed by a parallel reduction. contributions: 1) we design and implement a scalable $s u C A Q R$ solver that works efficiently for both tall and skinny and square matrices; 2) it demonstrates that we can simplify the software implementation by using a unique reduction tree meanwhile we can provide scalable performance; 3) we introduce an analytical model to accurately predict the optimal number of factorization domains by avoiding any tuning work, which is required by all existing CAQR implementations; and 4) detailed algorithm analysis and performance analysis reveal that the task-based dynamic scheduling approach is efficient in avoiding CPU idle cycles and hiding expensive communications, and should be adopted into more parallel software.

The rest of the paper is organized as follows. Next section introduces the performance issues in the existing distributed tiled CAQR solver. Section III illustrates the algorithm used by the $s u C A Q R$ solver. Section IV analyzes $s u C A Q R$ with respect to both computation and communication costs. Section $\mathrm{V}$ presents an analytical model to predict the optimal number of domains. Section VI describes $s u C A Q R$ 's dynamic dataflow execution within TBLAS and Section VII shows the experimental results. The final two sections present the related work and summarize this work.

\section{BACKGROUND}

This section briefly introduces the previous work of distributed tiled CAQR [9] and its existing performance bottleneck.

Given a matrix $A$ with $m_{b} \times n_{b}$ blocks $\left(m_{b}>n_{b}\right)$, the distributed tiled CAQR divides the matrix into a number of groups of rows. Each group is called a domain. For instance, $m_{b}$ rows will be cut into $D$ (horizontal) domains. Assuming $P$ MPI processes are created, each process will be responsible for a number of $\frac{D}{P}$ consecutive domains. Each domain corresponds to a local $\mathrm{QR}$ factorization and requires no communication. The results from all the domains must be merged (or reduced) to a single result. To implement the global parallel reduction, two level of trees are deployed: domainslevel reduction tree and inter-process reduction tree.

However, the distributed tiled CAQR algorithm cannot scale well if the matrix is not tall and skinny. Given a matrix with $m_{b}$ rows of tiles and $P$ processes, each process only computes its own $\frac{m_{b}}{P}$ rows. As the algorithm visits and computes the matrix from top left to bottom right, more processes become idle, which results in load imbalance and poor performance. If the number of columns $n_{b}$ is greater than $\frac{m_{b}}{P},\left\lceil n_{b} /\left(\frac{m_{b}}{P}\right)\right\rceil-1$ processes will become idle at the end of the computation. For instance, if $m_{b}=n_{b}, P-1$ processes will become idle at the last iteration of the algorithm. In this paper, we design a more general $\mathrm{QR}$ factorization solver that can achieve high scalability for all matrix shapes.

\section{ThE Algorithm}

suCAQR essentially consists of three components: 1) the tile data storage, 2) the logical/physical data layouts, and 3) the suCAQR computation. After a matrix is distributed to different processes, the data can be viewed from two perspectives: a 
logical data layout and a physical data layout. The rest of the section describes the three components.

\section{A. Tile Data Storage}

suCAQR uses a tile data storage. We divide a matrix into $b \times b$ square blocks (also known as tiles). Each tile is stored in a contiguous memory block. If the tile size is tuned appropriately, we can fit multiple tiles in caches and maximize the cache hit rate. For instance, a $12 \times 12$ matrix can be represented as follows:

$$
\left(\begin{array}{ccc}
a_{1,1} & a_{1,2} & a_{1,3} \\
a_{2,1} & a_{2,2} & a_{2,3} \\
a_{3,1} & a_{3,2} & a_{3,3}
\end{array}\right), \text { where }
$$

$a_{i, j}$ represents a tile of size $4 \times 4$. In the algorithm, every computational kernel takes as input several individual tiles (i.e., the basic unit) and computes new output.

Given an input matrix with $m_{b} \times n_{b}$ tiles, $s u C A Q R$ allocates a 2-D $m_{b} \times n_{b}$ array of pointers, each of which points to a memory block that stores a distinct tile.

\section{B. Usage of a Mixed Logical/Physical Layout}

We use a block-cyclic distribution method to distribute $m_{b}$ rows of tiles to $P$ processes. The method first divides all the tile rows into groups of size $B$, then distributes the groups to different processes. With the block-cyclic distribution method, the $i$-th tile row is stored in process $\frac{i}{B} \bmod P$. After the distribution, each process has $\frac{m_{b}}{P}$ rows of tiles.

As shown in Figure 3. (a), twelve rows of a matrix are distributed to four processes $\left(P_{0}, P_{1}, P_{2}\right.$, and $\left.P_{3}\right)$ in a blockcyclic way, where the group size $B=1$. We call this data layout a logical (or "virtual") data layout. However, physically, each process stores three virtually interleaved rows together in its local memory. Figure 3. (b) shows the corresponding physical data layout where each process owns three rows. Note that $s u C A Q R$ uses both physical layout and logical layout. While most parallel solvers only need to use the physical data layout, $s u C A Q R$ must follow the logical cyclic

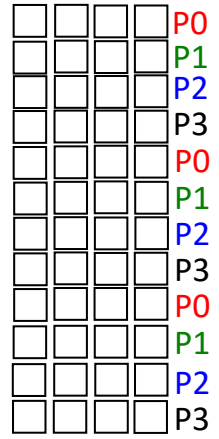

(a) Logical data layout

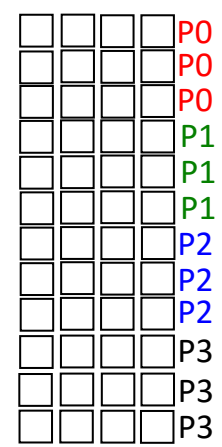

(b) Physical data layout
Fig. 3. After distributing a matrix with $12 \times 4$ tiles to four processes in a block cyclic way, we can view the same matrix from two perspectives: a logical data layout and a physical data layout. Both the logical data layout and the physical data layout are utilized by the $s u C A Q R$ algorithm. pattern (e.g., $P_{0}-P_{3}, P_{0}-P_{3}, \ldots$ ) such that the trailing submatrices consistently involve all the processes to achieve load balancing. It might appear that only one layout is needed here, but two types of computations working on two different layouts require that we deal with both of them.

As detailed in next subsection, suCAQR applies local CAQR factorizations to the physical data layout and applies interprocess binary tree reduction to the logical data layout at each iteration. To keep track of both physical data layout and logical data layout, we use the subroutine of physical_2_logical (as shown in Algorithm 1) to translate a row index from a physical data layout to a logical data layout (similar to the virtual memory idea used in operating systems).

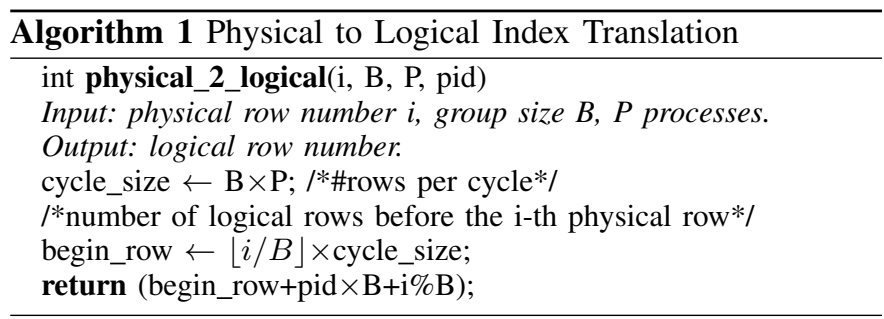

\section{C. suCAQR Computation}

The parallel implementation of $s u C A Q R$ is shown in Algorithm 2. Given a matrix with $m_{b} \times n_{b}$ tiles, the algorithm goes through $n_{b}$ iterations. At each iteration, there are two stages of computations: 1) every process performs a local CAQR factorization independently, followed by 2) a parallel binarytree reduction to merge the partial results from stage 1 to get the final result.

Stage 1: In the first stage (lines 3-9), every process decides the beginning row that has not computed the final result in the physical data layout. As the iteration number increases, more and more rows in the top portion of each process get the final result. Next, each process computes a local CAQR factorization on its local matrix, which spans from the beginning row to the last local row. The subroutines to determine the beginning row and perform local CAQR are briefly introduced as follows, respectively.

- get_first_row_position: In Algorithm 3, given a column number $k$, it first checks which process the tile $[k, k]$ belongs to (i.e., the root process root_pid). Depending on the relative position of the current process to the root process (e.g., above, same, below), the first row that has not computed result may lie in one of the three different locations (corresponding to the three conditional statements in Algorithm 3), respectively.

- local_caqr: Algorithm 4 calls six basic computational kernels, which are dgeqrt, dormqr, dtsqrt, dtsssmqr, dttqrt, and dttssmqr. To simplify our illustration, we will use the notations of QR1, UP 1 (stands for update), QR2, UP2, Merge, and MergeUpdate to represent the six kernels correspondingly. The local CAQR factorization will be applied to the submatrix whose local rows are between phys_1st_row and the 


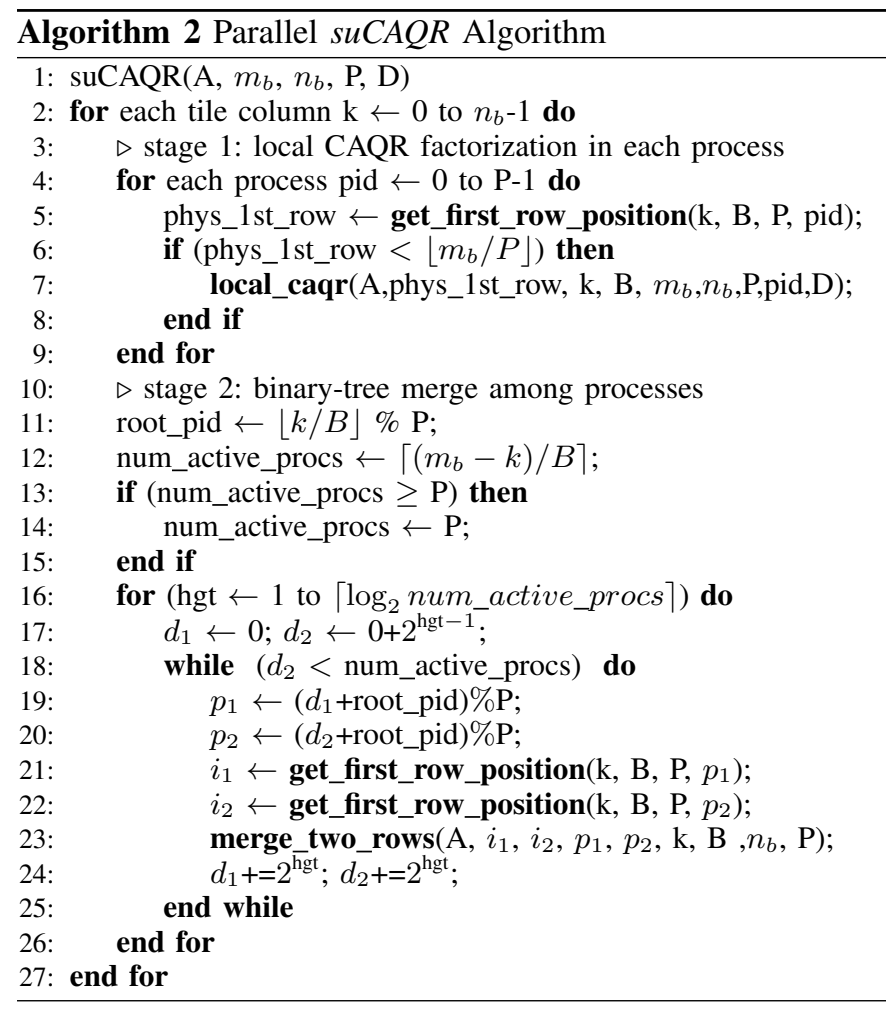

$\left(m_{b} / P\right)$-th row, and whose columns are between the $k$-th column and the $n_{b}$-th column. The local submatrix can be regarded as $D$ domains of rows. The parameter $D$ is an argument passed to the solver, which can vary from one to the number of rows per process. Local CAQR first computes a partial result for each domain, and then summarizes the results by using a local binary-tree merge. The computations from the $D$ domains can be executed in an embarrassingly parallel way. Section $\mathrm{V}$ introduces how to determine the optimal number of domains.

Stage 2: In the second stage (lines 10-26 in Algorithm 2), a parallel binary-tree reduction operation is conducted by a number of processes. It first decides the root of the parallel reduction tree, which changes in a block-cyclic manner. Next, it decides which processes are involved in computing the trailing submatrix (lines 12-15). We refer to the involved processes as active processes. The set of active processes will participate in the parallel reduction operation and each process contributes one row of tiles. The binary tree has a height of $\left\lceil\log _{2}\right.$ (ActiveProcesse $\left.)\right\rceil$. It merges the results from all the leaves up to the proper root identified by root_pid. Subroutine merge_two_rows is responsible for merging the partial results from two processes. As shown in Algorithm 5, it merges the $i_{1}$-th row in process $p_{1}$ and the $i_{2}$-th row in process $p_{2}$ to obtain a new result.

Dynamic Trees: The parallel reduction operation is a global operation that involves all the active processes. Since the suCAQR factorization proceeds from the top left corner to the bottom right corner of the matrix, and due to the block cyclic data layout, the root of the binary tree must change in a block
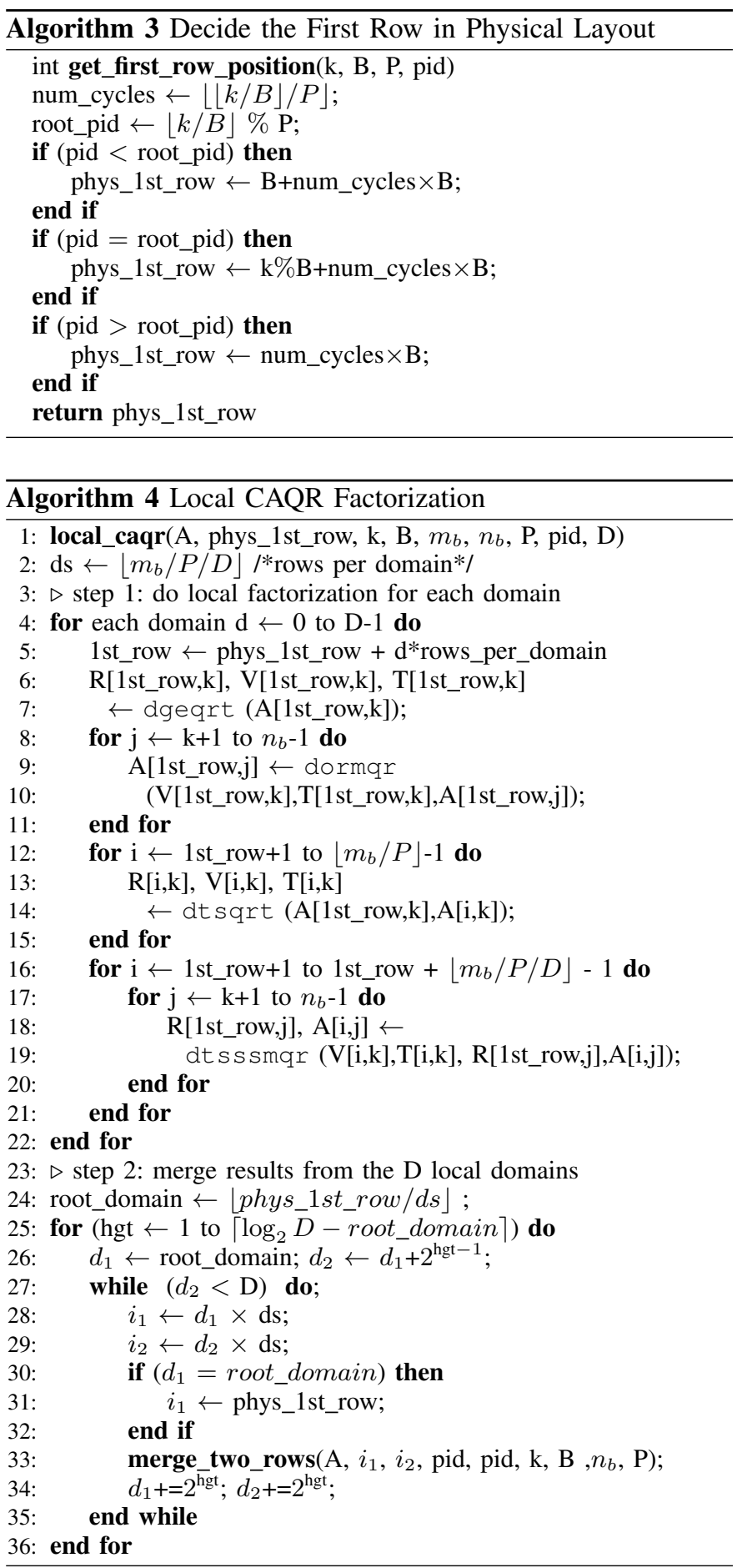

cyclic way. Figure 4 shows how the root of a parallel reduction tree may change dynamically. Suppose a matrix is distributed to four processes (P0, P1, P2, P3), the four processes need to participate in a global parallel reduction at every iteration. Each parallel reduction tree has a different root and the root changes in a round robin manner. If there are more than four iterations, the same pattern will be repeated for multiple times.

An Example: We use Figure 5 to show a simple example of the parallel $s u C A Q R$ algorithm. In Figure 5. (a), the input 


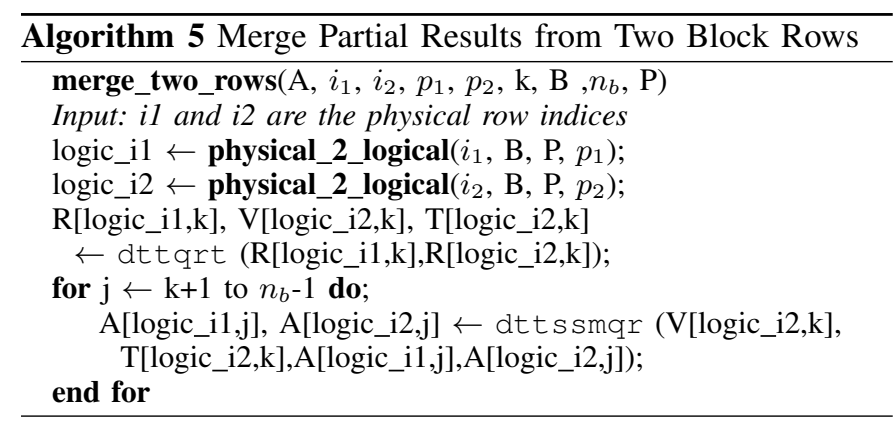

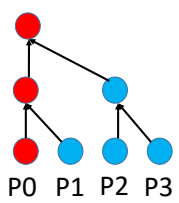

1st iteration

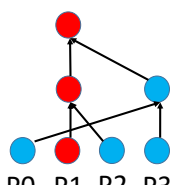

P0 P1 P2 P3

2nd iteration

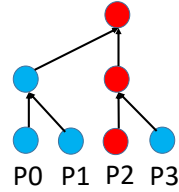

3rd iteration

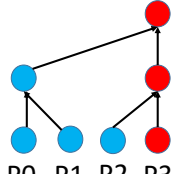

$\begin{array}{llllllll} & \text { P0 } & \text { P2 } & \text { P3 }\end{array}$
Fig. 4. An example of the parallel reduction operation. Given a matrix with four columns and four processes, suCAQR performs the global parallel reduction operation four times (once per iteration). Each iteration corresponds to a different parallel tree with a different root (e.g., P0 is the root (shown in red) in 1st iteration, P1 is the root (shown in red) in 2 nd iteration, etc.).

matrix has $12 \times 4$ tiles and is distributed to $\mathrm{P} 0-\mathrm{P} 3$ using a block cyclic distribution method, where the group size B equals one and the number of domains per process equals one. Figure 5. (b) shows the physical data layout of the matrix, where each process stores three rows from three separated rows from subfigure (a). At the first iteration, in Figure 5. (b), every process performs a local CAQR factorization. The local factorization applies QR1 and QR2 to the first column, applies UP1 to all tiles on the first row, and applies UP2 to all tiles on the trailing submatrix. All processes are performing the same computation in parallel but on different data.

After local factorizations are completed, the global binarytree reduction will start. Since the binary tree reduction is based on a logical data layout, Figure 5. (c), (d), and (e) use the logical data layout to display the matrix. After (b), all processes have obtained their partial $\mathrm{R}$ factors which are located on their first rows. Figure 5. (c) shows the status of the binary tree at the bottom level (i.e., depth=2), where P0 and P1 merge results meanwhile P2 and P3 merge results. Figure 5. (d) shows the second level of the binary tree (i.e., depth=1), where $\mathrm{P} 0$ and $\mathrm{P} 2$ merge results and store the final result to the root process $\mathrm{P} 0$.

As shown in Figure 5. (e), the second iteration will start with a trailing submatrix with one less row and one less column. In the second iteration, P1 becomes the root of the parallel reduction tree, and the same steps will be repeated on the smaller trailing submatrix.

\section{PARallel Algorithm Analysis}

This section analyzes the time complexity and communication cost of $s u C A Q R$. In the analysis, we assume the tile size is $b$, and the matrix is of $m_{b} \times n_{b}$ tiles. There are $P$ processes and each process has a number of $D$ domains. Note that our analysis is more general than the analysis of distributed tiled CAQR, which assumes $m_{b}>>n_{b}$.

\section{A. Operation Count per Process}

First, we compute the number of operations per process as follows:

QR1 (dgeqrt): $2 b^{3}$ operations per task. At each iteration, every domain of the process has one $Q R I$ task. Thus,

$$
T_{\text {dgeqrt } / \text { proc }}=\sum_{k=1}^{n_{b}} D \times\left(1 \times 2 b^{3}\right)=2 b^{2} n D .
$$

UP1 (dormqr): $3 b^{3}$ operations per task. At iteration k, every domain contains $\left(n_{b}-k\right) U P l$ tasks. Thus,

$$
T_{\text {dormqr } / \text { proc }}=\sum_{k=1}^{n_{b}} D \times\left(n_{b}-k\right) \times 3 b^{3} \approx \frac{3}{2} n^{2} b D .
$$

QR2 (dtsqrt): $\frac{10}{3} b^{3}$ operations per task. At iteration k, every domain has $\left(\left(m_{b}-k+1-P\right) / P-D\right) Q R 2$ tasks. Thus,

$$
\begin{aligned}
T_{d t s q r t / p r o c} & =\sum_{k=1}^{n_{b}}\left[\left(m_{b}-k+1-P\right) / P-D\right] \times \frac{10}{3} b^{3} \\
& \approx \frac{10}{3 P} b\left(m n-\frac{n^{2}}{2}-P n b D\right) .
\end{aligned}
$$

$U P 2$ (dtsssmqr): $4 b^{3}+s b^{2}$ operations per task. $s$ is used as the size of inner tile in a $b \times b$ tile. At iteration $\mathrm{k}$, each domain has $\left[\left(m_{b}-k+1-P\right) / P-D\right] \times\left(n_{b}-k\right)$ tasks. Thus,

$$
\begin{aligned}
T_{d t s s s m a r / p r o c}= & \sum_{k=1}^{n_{b}}\left[\left(m_{b}-k+1-P\right) / P-D\right] \\
& \times\left(n_{b}-k\right) \times\left(4 b^{3}+s b^{2}\right) \\
\approx & \frac{4+\frac{s}{b}}{P}\left(\frac{n^{2} m}{2}-\frac{n^{3}}{6}-\frac{n^{2} b P}{2} D\right) .
\end{aligned}
$$

Merge (dttqrt): $\frac{5}{3} b^{3}$ operations per task. At iteration k, each process executes it for $(\log P+D-1)$ times. Thus,

$$
\begin{aligned}
T_{d t t q r t / p r o c} & =\sum_{k=1}^{n_{b}}(\log P+D-1) \times\left(\frac{5}{3} b^{3}\right) \\
& =\frac{5}{3} b^{2} n(\log P+D-1) .
\end{aligned}
$$
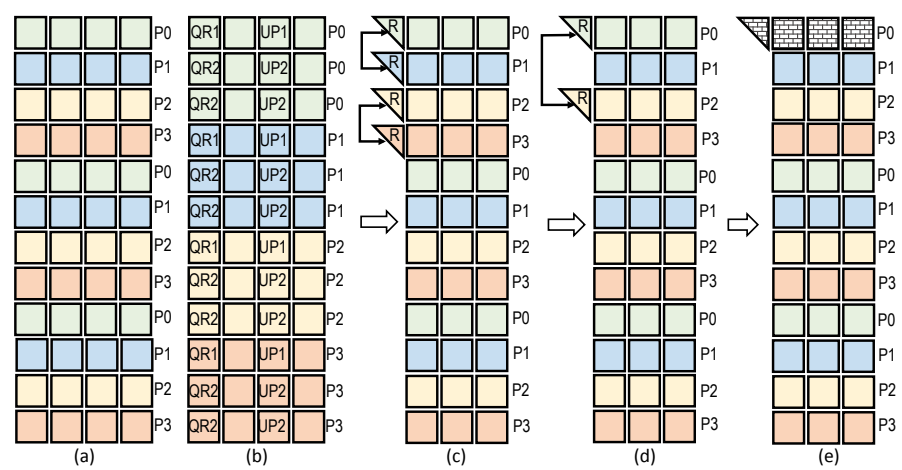

Fig. 5. An example of the parallel $s u C A Q R$ algorithm given a matrix with $12 \times 4$ tiles and four processes. Different processes are denoted by different colors. (a) Input matrix in a logical data layout. (b) Physical data layout and corresponding local CAQR factorization in each process. (c) Merging in the third level of the binary tree. (d) Merging in the second level of the binary tree. (e) Matrix status at the beginning of the second iteration. 
TABLE I

COMPARISON WITH OTHER QR FACTORIZATIONS FOR $n \times n$ MATRICES

\begin{tabular}{|c|c|c|c|}
\hline & Op Count (parallel) & \#Messages & \#Words \\
\hline Lower bound & $\Theta\left(\frac{n^{3}}{P}\right)$ & $\Theta(\sqrt{P})$ & $\Theta\left(\frac{n^{2}}{\sqrt{P}}\right)$ \\
\hline Scalapack [4] & $\frac{4 n^{3}}{3 P}$ & $\frac{5 n}{4} \log ^{2} P$ & $\left(n^{2}+b n\right) \log P$ \\
\hline $\begin{array}{c}\text { distributed } \\
\text { tiled CAQR [9] }\end{array}$ & $\begin{array}{c}\frac{n^{3}}{3 P}\left(6+\frac{s}{2 b}\right) \\
+\frac{n^{2} b}{4}\left(4+\frac{s}{b}\right) \log P \\
-\frac{n^{2} b}{2}\left(3+\frac{s}{b}\right)\end{array}$ & $\left(\frac{n}{b}\right)^{2} \log P$ & $n^{2} \log P$ \\
\hline$s u C A Q R$ & $\begin{array}{c}\frac{n^{3}}{3 P}\left(4+\frac{s}{b}\right) \\
+\frac{n^{2} b}{4}\left(4+\frac{s}{b}\right) \log P \\
-\frac{n^{2} b}{2}\left(3+\frac{s}{b}\right) \\
+(D-1) n\left(\frac{b^{2}}{3}+s n\right)\end{array}$ & $\left(\frac{n}{b}\right)^{2} \log P$ & $n^{2} \log P$ \\
\hline
\end{tabular}

MergeUpdate (dttssmqr): $\frac{1}{2}\left(4 b^{3}+s b^{2}\right)$ operations per task. At iteration $\mathrm{k}$, each process executes it for $(\log P+$ $D-1)\left(n_{b}-k\right)$ times. Thus,

$$
\begin{aligned}
T_{d t s s m q r / \text { proc }} & =\sum_{k=1}^{n_{b}}(\log P+D-1)\left(n_{b}-k\right) \times \frac{1}{2}\left(4 b^{3}+s b^{2}\right) \\
& \approx n^{2}\left(b+\frac{s}{4}\right)(\log P+D-1) .
\end{aligned}
$$

\section{B. Communication Cost}

We consider the process that has the maximum of messages. Since our calculation is the same as that of distributed tiled $\mathrm{CAQR}$, we simply present the conclusion here: \#messages $=$ $\log _{2}(P) \frac{n^{2}}{b^{2}}$, and $\#$ words $=\log _{2}(P) n^{2}$.

\section{Comparison with the Related Work}

We compare $s u C A Q R$ with the theoretical lower bound, ScaLAPACK, and the distributed tiled CAQR with respect to the operation count, the number of messages, and the number of words communicated. As displayed in Table I, suCAQR has less operations than distributed tiled CAQR if its number of domains $D$ equals one, but has more operations than ScaLAPACK by a fraction of $\frac{s}{4 b}$. Note that $s$ is typically much less than $b$. Both distributed tiled CAQR and suCAQR have more messages than ScaLAPACK. This is because they are designed to send and receive small messages of tiles to minimize a process's CPU idle time by increasing the degree of parallelism. As later shown in Section VI, our implementation of asynchronous execution is able to hide the communication latency by a great number of fine-grain tasks.

\section{Deciding Optimal Number of Domains}

Algorithm 4 defines that each process can have $D$ domains, where $D$ is an integer between one and $\frac{m_{b}}{P}\left(D=\frac{m_{b}}{P}\right.$ means that every title row is a domain). Based on our experiments, the number of domains has a significant impact on the program performance. Figure 6 shows that a different $D$ may lead to totally different performance on a given matrix.

In order to determine an optimal number of domains without a lot of tuning and searching efforts, we analyze the algorithm and make the following observations:
1) Within one domain, the degree of task parallelism is decided by the number of block columns $n_{b}$. That is, each domain can contain up to $n_{b}$ parallel tasks (e.g., there are $n_{b}-k$ parallel tasks in the $k$-th iteration).

2) When $n_{b}$ is much greater than the number of threads per process (i.e., $T$ ), using one domain can attain the minimum number of operations, the maximum number of higher-performance UP2 tasks, and thus the best performance.

3) Whenever $D$ increases, the degree of task parallelism within each process increases by $D$ times. However, when $D$ increases a lot, the number of the fastest UP 2 tasks starts to decrease and the overall performance will degrade instead.

Based on the analysis, we develop a novel model to determine the optimal number of domains. First, we make sure the degree of task parallelism within a process must be greater than the number of threads per process $(T)$. This relationship can be expressed as follows:

$$
\begin{gathered}
\text { (degree } \left._{\text {parallel }}=n_{b} \times D\right) \geq c \times T \\
\Longrightarrow D \geq\left\lceil\frac{c \times T}{n_{b}}\right\rceil
\end{gathered}
$$

We choose coefficient $c=4$ based on our collected performance data. As long as $n_{b} \geq 4 T$, using one domain can produce the best performance regardless of the matrix shape being skinny or square. Also, when $n_{b}$ becomes less and less, the formula allows $D$ to increase correspondingly to compensate for the diminishing degree of task parallelism. When $n_{b}$ becomes too small (e.g., 1, 2, or 3 columns), we set $D=T$ to let $T$ threads compute $T$ domains in an embarrassingly parallel way. Further creating more domains than the number of threads per process will slow down the thread performance due to increased operations, fewer fast UP 2 tasks, and an increment in the working set from irregular cross-domain merges.

The complete model is provided as below, where $c=4$.

$$
D^{*}= \begin{cases}\left\lceil\frac{c \times T}{n_{b}}\right\rceil & \text { if } n_{b} \geq c \\ \min \left\{T, \frac{m_{b}}{P}\right\} & \text { if } n_{b}<c\end{cases}
$$

Figure 6. (a) shows the actual performance using different number of domains, and the performance using the predicted $D^{*}$ based on the analytical model. We execute 4 processes and each process consists of 16 threads. The input matrix size goes from $128 \times 1$ blocks to $128 \times 128$ blocks. For each matrix, we try all the possible number of domains and collect their performance. From the data, we can see that our model-based prediction matches the actual best number of domains closely. In the second experiment, we take the same matrix size of $128 \times 16$ blocks but change the number of threads per process from one to 32. As shown in Figure 6. (b), the model-based prediction again has a good match to the actual best number of domains. In the following experiments displayed in Section VII, we have also observed that the actual best number of domains matches the analytical model. 


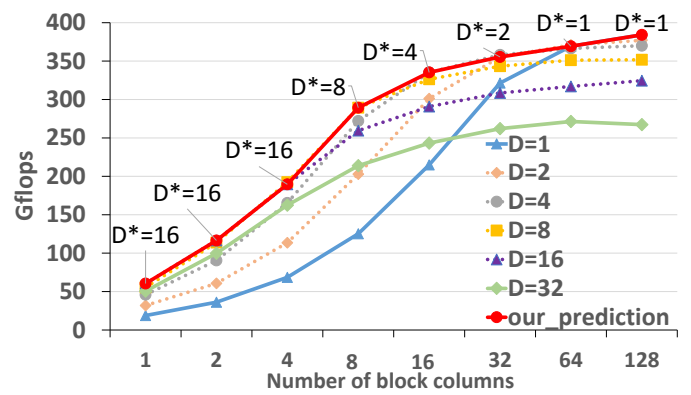

(a) The number of block columns changes

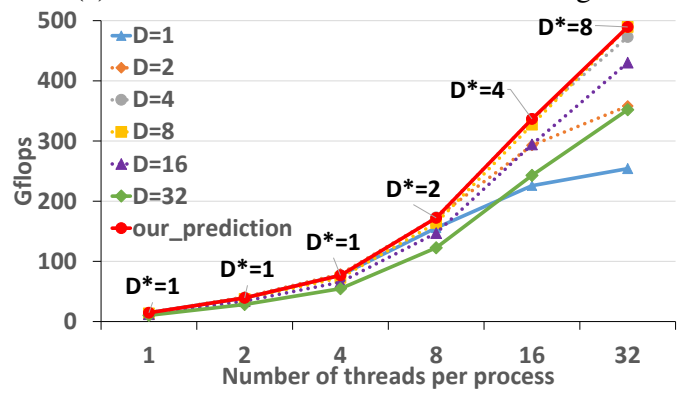

(b) The number of threads per process changes

Fig. 6. Using an analytical model to predict the optimal number of domains $D^{*}$. In (a), each matrix has 128 block rows but different block columns. Each process has 16 threads. In (b), the input matrix has 128 block rows and 16 block columns but uses a different number of threads.

\section{ThE ASYNCHRONOUS DATAFLOW IMPLEMENTATION}

The parallel algorithm of $s u C A Q R$ has been implemented with the TBLAS [11] runtime system. The TBLAS runtime system is able to support distributed and dynamic DAG scheduling using all CPU cores. It consists of three types of threads: task-generation thread, compute thread, and communication thread. The task-generation thread executes a taskbased $s u C A Q R$ program and generates fine-grain tasks to fill in multiple priority-based task queues. Whenever becoming idle, a compute thread picks up a ready task from the ready task queue and executes it. The communication thread is responsible for sending and receiving data between a parent task and its children to satisfy the data dependency requirement. The major distinction of TBLAS is that it does not require representing or constructing a task graph by users but dynamically solves data dependencies among all distributed compute nodes.

With TBLAS, our implementation work is to write a sequential program to generate tasks that are needed in the $s u C A Q R$ algorithm. The rest of the parallel implementation is handled by the TBLAS runtime system, which can automatically detect data dependencies by simply matching a task's output to another task's input on the fly, and schedule tasks to CPUs dynamically. In the $s u C A Q R$ program, we create six types of tasks: QR1, QR2, UP1, UP2, Merge, and Merge Update. Each type of task takes multiple tiles as input and writes new result to the output tiles. To execute the $s u C A Q R$ program, we launch a number of MPI processes on different multicore compute nodes. Every MPI process is managed by one TBLAS runtime system. The runtime systems coordinate with each other to

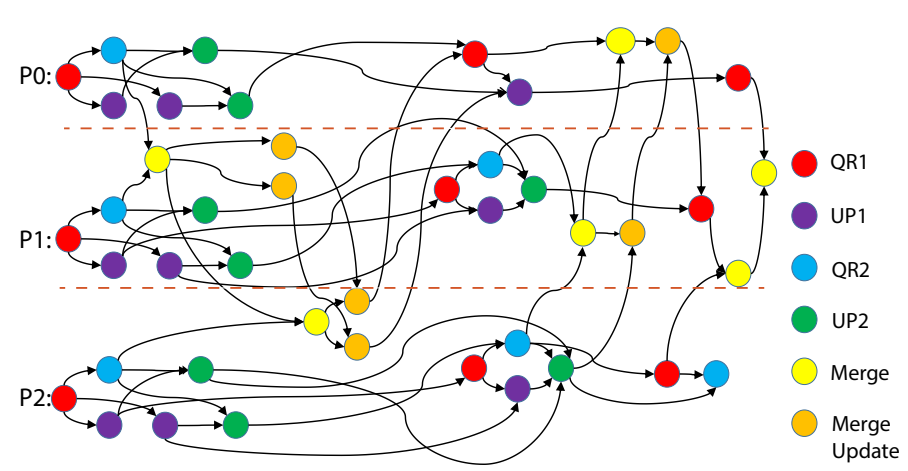

Fig. 7. The corresponding DAG of the $s u C A Q R$ algorithm computing a matrix of $6 \times 3$ tiles with 3 processes. Six tile rows are distributed to the three processes in a cyclic way such that each process has two rows of tiles.

solve data dependencies, dispatch tasks, and send the output of a parent task to its children tasks which are waiting for their input.

The execution of the task-based $s u C A Q R$ program is driven by data availability. When the task-based program is being executed, the frontier portion of the DAG is unrolled dynamically by the runtime system. The size of the active DAG is controlled by a task window size. Each runtime system has its own execution point, which follows the data-availability path to reach a different place in the DAG. Figure 7 shows the complete DAG of $s u C A Q R$ which solves a matrix of $6 \times 3$ tiles. The task graph is executed by three processes, each of which stores two rows of tiles. From the figure, we can see that three processes execute from the left to the right and communicate with each other only when it is necessary. Note that there is no global synchronization point in the execution, and no artificial order between tasks except for the real data dependencies.

\section{EXPERIMENTAL RESULTS}

We first evaluate the impact of tile size on $s u C A Q R$ 's computational kernels, and compare $s u C A Q R$ with the distributed tiled CAQR to show the performance improvement. Next, we present the breakdown of the total execution time to analyze the overhead of different components in our implementation. Finally, we compare $s u C A Q R$ with ScaLAPACK and DPLASMA [12] in weak scalability. For DPLASMA, we have tuned and selected the best tree type and the best $D$. Both $s u C A Q R$ and DPLASMA use the tuned tile size $\mathrm{b}=300$.

We conducted experiments on the Big Red II Cray XE6/XK7 supercomputer [13] at Indiana University. Table II shows the specifications of the computer system. Each compute node has 32 cores and 64 GB of memory, and runs a Cray Linux OS. Since the mathematics library of Cray LibSci provides a faster performance than Intel MKL (see Figure 8), we use Cray LibSci to run the experiments.

\section{A. Impact of Tile Size on Sequential Computational Kernels}

In our implementation, the tile size plays an important role in the overall performance. To search for the best tile size, we test a number of tile sizes on the sequential computational 


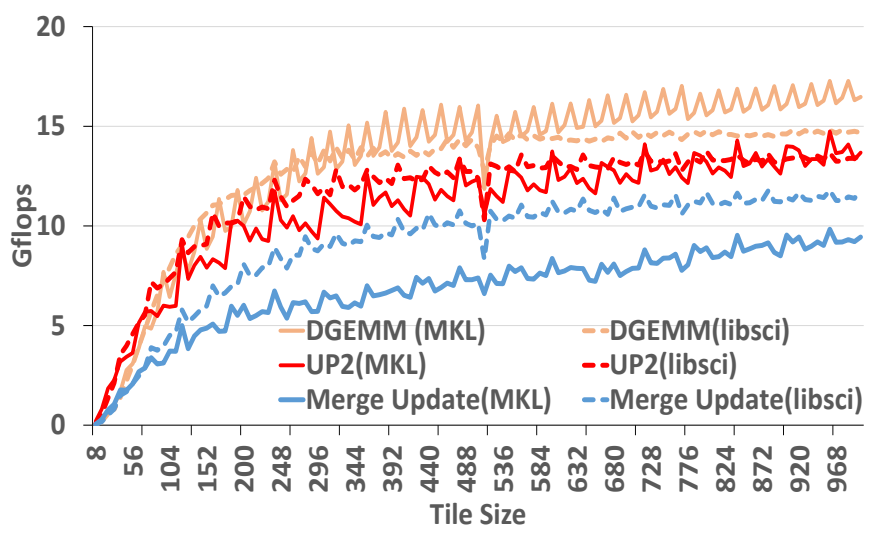

Fig. 8. Performance of sequential suCAQR kernels with different tile sizes using Intel MKL and Cray LibSci. DGEMM implies a practical upper bound.

kernels. Figure 8 shows the performance of the UP 2 and MergeUpdate kernels in $s u C A Q R$, as well as DGEMM provided by Cray LibSci and Intel MKL. DGEMM demonstrates the practical maximum performance on the computer system. From the figure, we can see that the performance of the kernels increases as the tile size becomes larger. In general, we want to have a bigger tile size to maximize the kernel performance. However, a bigger tile size results in less number of tasks and degraded load balance. Hence, a good tile size is often a number between 200 and 400. In our implementation, we choose 300 as the tile size.

The other important observation is that the kernel of UP 2 is much faster than the kernel of MergeUpdate. As the number of domains per process $(D)$ increases, the number of MergeUpdate tasks will increase. As a result, the overall performance will drop. This is one of the reasons that we do not want $D$ to be large unless the degree of parallelism is too small.

\section{B. Comparison with Previous Distributed Tiled CAQR}

We compare $s u C A Q R$ with distributed tiled CAQR to evaluate the performance gain of our new solver. In the experiment, we use 512 cores and fix the number of rows for the input

TABLE II

SPECIFICATIONS OF THE EXPERIMENTS

\begin{tabular}{l|l}
\hline OS & Cray Linux Environment \\
\hline Nodes & 1,020 (a maximum of 2048 cores per job) \\
\hline Memory per node & $64 \mathrm{~GB}$ \\
\hline Processors per node & 2 \\
\hline Cores per processor & 16 \\
\hline FPU info & two cores share one FPU \\
\hline Processor & AMD Opteron 6380 2.5GHz \\
\hline Peak performance/core & 13.6 Gflops (with AMD turbo core) \\
\hline MPI & Cray-MPICH 7.2.5 \\
\hline suCAQR & linked with Cray LibSci 13.2 \\
\hline ScaLAPACK & Cray LibSci 13.2 \\
\hline DPLASMA's HQR & $\begin{array}{l}\text { PaRSEC / DPLASMA 2.0.0, PLASMA 2.7.1, } \\
\text { linked with Cray LibSci 13.2, } \\
\text { Tuned and selected the best tree } \\
\text { and D (between 1 and 4), tile size b=300. }\end{array}$ \\
\hline \multicolumn{2}{|l}{}
\end{tabular}

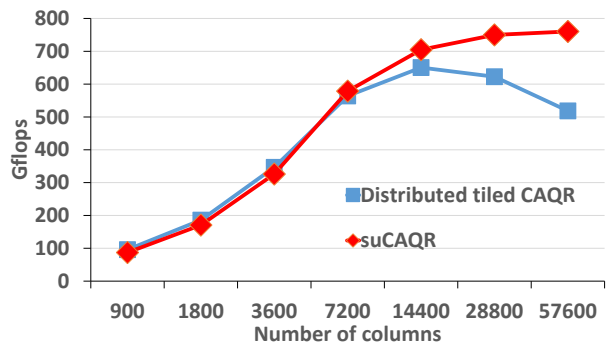

Fig. 9. Comparison of suCAQR with distributed tiled CAQR. All the input has a fixed number of 57,600 rows but a varying number of columns.

matrices. Each matrix is of dimension $57,600 \times n$, where $n$ is increased from 900 to 57,600. Figure 9 shows the performance of distributed tiled CAQR and $s u C A Q R$. When $n$ is greater than or equal to 14,400 (i.e., $\frac{\# \text { columns }}{\# \text { rows }}=\frac{1}{4}, \frac{1}{2}$, and 1), suCAQR starts to be faster than distributed tiled CAQR.

The reason can be illustrated by a simple example shown in Figure 10. The example shows how the tiled CAQR algorithm and the $s u C A Q R$ algorithm would use four processes to factor an $8 \times 5$ matrix, respectively. In Figure 10. (a), P0 becomes idle after two iterations as shown in (2), then P1 also becomes idle after another two iterations as shown in (3). Note that half of the processes have no work to do in (3). In Figure 10. (b), P0-P3 are kept busy all the time from the first iteration to the last iteration.

\section{Runtime Efficiency Analysis}

To analyze the runtime efficiency, we measure the time spent on each component of the suCAQR implementation. As presented in Section VI, our implementation uses one generation thread to i) create new tasks, one communication thread to ii) send/receive messages, and a number of compute threads to iii) get ready tasks, iv) execute the ready tasks, and v) fire new tasks that are waiting for the completed tasks. We

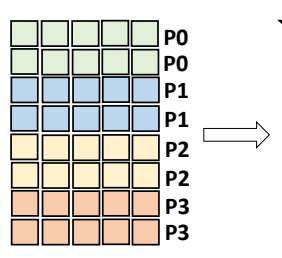

(1)

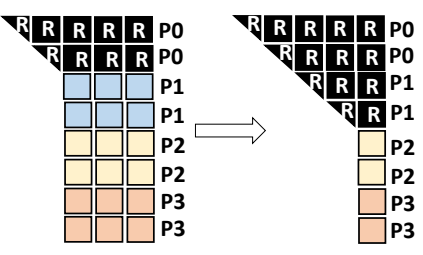

(3) (a) Distributed tiled CAQR

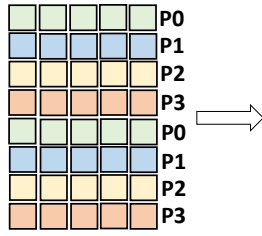

(1)

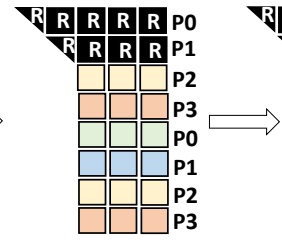

(2) (b) The new $s u C A Q R$

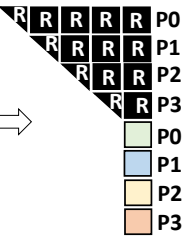

(3)
Fig. 10. (a) shows that more and more processes become idle as distributed tiled CAQR proceeds. (b) shows that there is no idle process in the new suCAQR algorithm. Black color represents the completed result. 


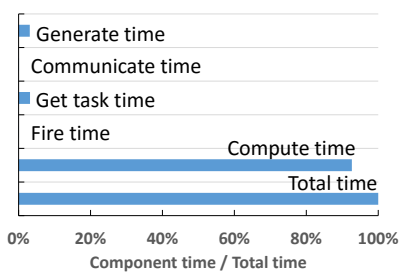

(a) extremely tall and skinny

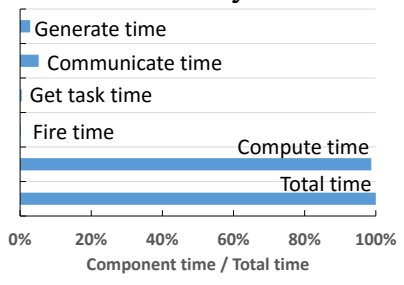

(c) $\frac{\# \text { columns }}{\# \text { rows }}=\frac{1}{4}$

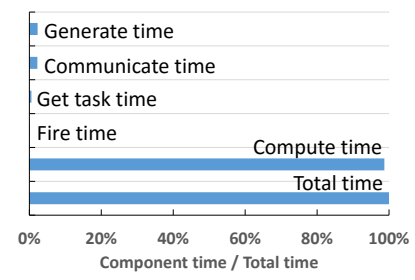

(b) $\frac{\# \text { columns }}{\# \text { rows }}=\frac{1}{16}$

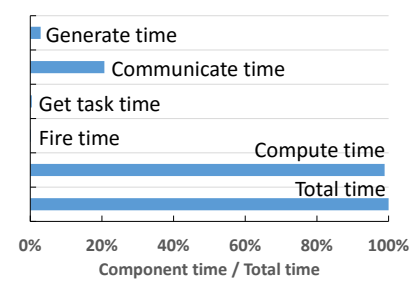

(d) square
Fig. 11. Breakdown of the total execution time. The total wall clock time is nearly equal to the compute time taken by the computational kernels.

instrument the source code to measure the time spent on each of the five components.

Figure 11 lists the breakdown of the total execution time using 512 cores. We consider four different matrix shapes for which the ratio of the number of columns to the number of rows is: extremely tall and skinny, $\frac{1}{16}, \frac{1}{4}$, and square, respectively. As shown in Figure 11, the percentages of the generate-task time, get-task time, and fire-task time are small from (a) to (d). Although the percentage of the communication time increases, it is hidden by a large number of dynamic scheduling computational tasks. For different matrices, the percentage of the compute time relative to the total clock time is $92.7 \%, 98.7 \%, 98.7 \%$, and $98.9 \%$, respectively. It implies that the wall clock time is almost equal to the CPU time that is taken by the computational kernels. Also, all the other costs including runtime overhead and communication only occupy at most $7 \%$ of the total time. Therefore, this result demonstrates the good efficiency of the $s u C A Q R$ solver.

\section{Performance Evaluation in Weak Scalability}

We use weak scalability to measure the capability of $s u$ $C A Q R$ to solve larger problems if a user has access to more CPUs. In the experiments, whenever we double the number of cores, we also double the total amount of computation accordingly. The number of matrix elements per core is thus kept as a constant in each experiment.

Figure 12 displays the metric of Gflops-per-core for four different matrix shapes using up to 1,024 cores. From all four subfigures, we can see that $s u C A Q R$ maintains good scalability with a nearly constant Gflops-per-core except for the $40 \%$ drop from one core to two cores. The reason for the performance drop is that two AMD CPU cores are sharing one floating point unit (FPU) by using the dual stream mode.

As shown in Figure 12. (a), when the matrix is extremely tall and skinny (having only 6 block columns but many block

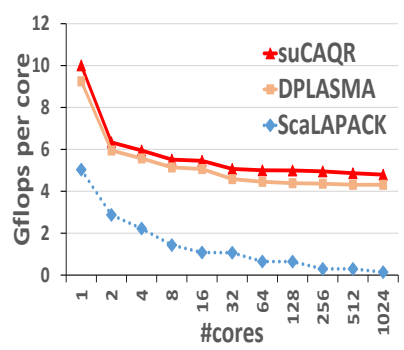

(a) extremely tall and skinny

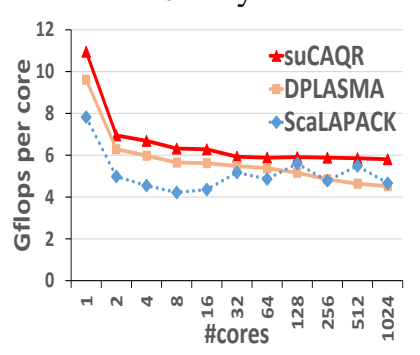

(c) $\frac{\# \text { columns }}{\# \text { rows }}=\frac{1}{4}$

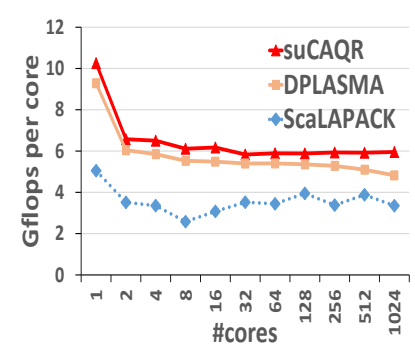

(b) $\frac{\# \text { columns }}{\# \text { rows }}=\frac{1}{16}$

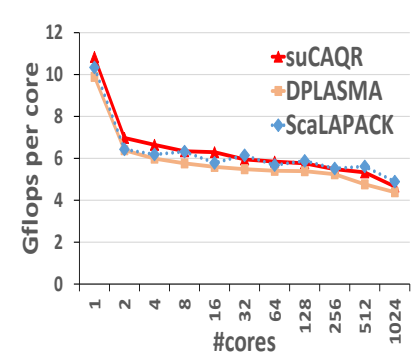

(d) square
Fig. 12. Experiments of weak scalability on 1024 cores.

rows), suCAQR can outperform ScaLAPACK by 30 times and outperform DPLASMA by $13 \%$ on 1024 cores. For the matrix shape of $\frac{1}{16}$, suCAQR outperforms ScaLAPACK by $78 \%$ and outperforms DPLASMA by 23\%. For the $\frac{1}{4}$ shape, suCAQR outperforms ScaLAPACK by $25 \%$ and outperforms DPLASMA by $30 \%$. When the matrix is purely square (in Figure 12. (d)), suCAQR is as good as ScaLAPACK, and is $6 \%$ better than DPLASMA.

We can also see that the performance of ScaLAPACK keeps rising from tall and skinny matrices to square matrices. This is because a wider matrix will produce a larger trailing submatrix which is more abundant with the vendor-optimized DGEMM kernel which is faster than $s u C A Q R$ 's kernels (see Figure 8). Another observation is that the performance of ScaLAPACK fluctuates a little bit when its 2-D process grid $P \times Q$ is not in a square shape (i.e., $P \neq Q$ ).

Reasons for the Performance Improvement: The $s u C A Q R$ is faster than DPLASMA due to the following reasons. First, we minimize the number of domains to maximize the number of invocations of the fastest kernel by using the analytical model of $D^{*}$. Also, less domains can result in fewer floating point operations (refer to Table I). Second, the single-domain's consecutive row-by-row update has a smaller working set size and better locality than multiple domains which work on multiple different rows. Third, the TBLAS runtime we use is efficient in hiding communication by computation such that the wall clock time is simply equal to the pure computation time, which is almost an ideal case. Furthermore, we modify DPLASMA to use the same binary tree and same parameters as $s u C A Q R$. The profiling results show that $s u C A Q R$ is still faster than DPLASMA owing to TBLAS's higher data reuse between tasks and the batched message passing. 


\section{RELATED WORK}

Different communication-avoiding algorithms have been applied to a variety of applications. Yelick et al. developed a communication avoiding GMRES solver on shared-memory multicore computers [14]. Grigori et al. designed the communication optimal LU factorization (CALU) algorithm [15]. Khabou et al. also designed a communication avoiding LU factorization version with panel rank revealing pivoting [16]. Ballard et al. created a communication avoiding algorithm for Strassen's matrix multiplication [17]. In this paper, we provide a faster parallel implementation of the CAQR algorithm with a simpler design, and is able to handle matrices of any shapes.

There are also other implementations of communication avoiding QR factorization that target different computing systems. Anderson et al. implemented the communication avoiding QR factorization entirely on a single GPU to achieve high performance [18]. Agullo et al. [19] developed a topologyaware approach that can adapt to a grid environment to compute the CAQR factorization by confining communications to local sites.

DPLASMA's HQR [10] studies different tree options such as flat tree, greedy tree, fibonacci tree, binary tree, and so on. DPLASMA also has an optional fourth level tree called domain-level tree. But it is not known when to include and how to optimize the fourth domain-level tree. Also, what type of tree is the best is totally dependent on the result of trying all the different trees at four different levels given a specific input and a certain number of CPUs. Differently, our work reveals that these types of complexities can be avoided without any loss of performance. In addition, we find that the optimal number of domains per process is only related to the number of block columns in the matrix and the number of CPU cores per compute node. We build an accurate analytical model to calculate the optimal number of domains conveniently. This knowledge is new and has not been discovered by other work.

\section{CONCLUSION}

Communication-avoiding QR factorization is a generic parallel algorithm and potentially has many different approaches to implementing and optimizing it. In this paper, we target the distributed multicore computer architecture and give a simple, efficient, and scalable design to handle various matrix shapes.

From the perspective of the algorithm, suCAQR uses a logical block cyclic data layout on which a dynamic-root parallel tree reduction method is deployed. It also uses a much simplified parallel reduction method to leverage the specific architectural strength of a cluster of multicore compute nodes. It is for the first time to provide an insight into determining the important factor of the number of domains without any tuning and searching. From the perspective of the software design, a simple unique binary tree can significantly simplify the software implementation and reduce the cost of parameter tuning. From the perspective of the implementation, suCAQR generates a large number of fine-grain tasks to achieve a high degree of parallelism, and allows for a fully dynamic execution to completely hide communication by computation using the dynamic scheduling TBLAS runtime. The experimental results have shown that $s u C A Q R$ can perform better than the state-ofthe-art QR factorization libraries on different matrices using up to 1,024 cores. The runtime efficiency analysis has also revealed a near optimal circumstance (i.e., wall clock time equals computation time) for efficient parallel executions.

\section{REFERENCES}

[1] E. Anderson, Z. Bai, and J. Dongarra, "Generalized QR factorization and its applications," Linear Algebra and its Applications, vol. 162, pp. 243-271, 1992

[2] A. Björck, Numerical methods for least squares problems. SIAM, 1996.

[3] J. W. Demmel, Applied numerical linear algebra. SIAM, 1997.

[4] L. S. Blackford, J. Choi, A. Cleary, E. D'Azevedo, J. Demmel, I. Dhillon, J. Dongarra, S. Hammarling, G. Henry, A. Petitet et al. ScaLAPACK users' guide. SIAM, 1997, vol. 4.

[5] J. Choi, J. Demmel, I. Dhillon, J. Dongarra, S. Ostrouchov, A. Petitet, K. Stanley, D. Walker, and R. C. Whaley, "ScaLAPACK: A portable linear algebra library for distributed memory computers: Design issues and performance," in Applied Parallel Computing Computations in Physics, Chemistry and Engineering Science. Springer, 1996, pp. 95106.

[6] J. W. Demmel, L. Grigori, M. F. Hoemmen, and J. Langou, "Communication-optimal parallel and sequential QR and LU factorizations," UTK, LAPACK Working Note 204, August 2008.

[7] G. Ballard, J. Demmel, L. Grigori, M. Jacquelin, N. Knight, and H. Nguyen, "Reconstructing householder vectors from tall-skinny QR," Journal of Parallel and Distributed Computing, vol. 85, pp. 3 - 31, 2015, IPDPS 2014 Selected Papers on Numerical and Combinatorial Algorithms.

[8] M. Hoemmen, "A communication-avoiding, hybrid-parallel, rankrevealing orthogonalization method," in 2011 IEEE International Parallel Distributed Processing Symposium (IPDPS), May 2011, pp. 966-977.

[9] F. Song, H. Ltaief, B. Hadri, and J. Dongarra, "Scalable tile communication-avoiding $\mathrm{QR}$ factorization on multicore cluster systems," in Proceedings of the 2010 ACM/IEEE International Conference for High Performance Computing, Networking, Storage and Analysis (SC'10), 2010, pp. 1-11.

[10] J. Dongarra, M. Faverge, T. Herault, M. Jacquelin, J. Langou, and Y. Robert, "Hierarchical QR factorization algorithms for multi-core clusters," Parallel Computing, vol. 39, no. 4, pp. 212-232, 2013.

[11] F. Song, A. YarKhan, and J. Dongarra, "Dynamic task scheduling for linear algebra algorithms on distributed-memory multicore systems," in Proceedings of the 2009 ACM/IEEE International Conference for High Performance Computing, Networking, Storage and Analysis (SC'09). IEEE, 2009, pp. 1-11.

[12] PaRSEC, "http://icl.utk.edu/parsec."

[13] BigRedII, "http://rt.uits.iu.edu/bigred2."

[14] M. Mohiyuddin, M. Hoemmen, J. Demmel, and K. Yelick, "Minimizing communication in sparse matrix solvers," in Proceedings of the Conference on High Performance Computing Networking, Storage and Analysis. ACM, 2009, p. 36.

[15] L. Grigori, J. W. Demmel, and H. Xiang, "CALU: A communication optimal LU factorization algorithm," SIAM Journal on Matrix Analysis and Applications, vol. 32, no. 4, pp. 1317-1350, 2011.

[16] A. Khabou, J. W. Demmel, L. Grigori, and M. Gu, "LU factorization with panel rank revealing pivoting and its communication avoiding version," SIAM Journal on Matrix Analysis and Applications, vol. 34, no. 3, pp. 1401-1429, 2013.

[17] G. Ballard, J. Demmel, O. Holtz, B. Lipshitz, and O. Schwartz, "Communication-optimal parallel algorithm for Strassen's matrix multiplication," in Proceedings of the twenty-fourth annual ACM symposium on Parallelism in algorithms and architectures, 2012, pp. 193-204.

[18] M. Anderson, G. Ballard, J. Demmel, and K. Keutzer, "Communicationavoiding QR decomposition for GPUs," in 2011 IEEE International Parallel \& Distributed Processing Symposium (IPDPS), 2011, pp. 4858.

[19] E. Agullo, C. Coti, J. Dongarra, T. Herault, and J. Langou, "QR factorization of tall and skinny matrices in a grid computing environment," in 24th IEEE International Parallel and Distributed Processing Symposium (IPDPS 2010). IEEE, 2010. 